\title{
Theaflavin-3,3'-digallate attenuates cigarette smoke extract-induced pulmonary emphysema in mice by suppressing necroptosis
}

\author{
GUANGXIN LUAN ${ }^{1,2 *}$, ZHEN ZHU ${ }^{2 *}$, KAIYUE WU ${ }^{1}$ and SHAOJUN YIN ${ }^{2}$ \\ ${ }^{1}$ National Demonstration Center for Experimental Fisheries Science Education, \\ Affiliated Shanghai Ocean University, Shanghai 201306; ${ }^{2}$ Department of Respiratory Medicine, \\ Affiliated Shanghai Sixth People's Hospital, Shanghai Jiao Tong University, Shanghai 200233, P.R. China
}

Received October 22, 2020; Accepted October 5, 2021

DOI: 10.3892/etm.2021.10933

\begin{abstract}
Pulmonary emphysema is one of the most important pathological manifestations of chronic obstructive pulmonary disease and is commonly associated with cigarette smoking. Previous studies have indicated that necroptosis, a novel non-apoptotic cell death mechanism associated with inflammation and oxidative stress, may contribute to the development of pulmonary emphysema. Theaflavin-3,3'-digallate (TF-3), one of the theaflavins present in black tea, is known to possess several bioactive properties. In the present study, it was demonstrated that TF-3 significantly reduced the generation of reactive oxygen species and the mRNA expression levels of TNF- $\alpha$, IL- $1 \beta$ and IL- 6 in CSE-treated human normal lung epithelial BEAS-2B cells. To further explore the role of TF-3 in necroptosis, the necroptotic rates of BEAS-2B cells were examined via flow cytometry and immunofluorescence assays. The results demonstrated that TF-3 may suppress necroptosis in CSE-treated BEAS-2B cells. Furthermore, it was determined that TF-3 significantly inhibited the CSE-induced phosphorylation of p38 MAPK, receptor-interacting serine/threonine-protein kinase three (RIPK3) and mixed lineage kinase domain-like (MLKL) in BEAS-2B cells. Another experiment demonstrated that a pharmacological inhibitor of the p38 MAPK pathway, SB203580, significantly reduced the protein expression levels
\end{abstract}

Correspondence to: Dr Shaojun Yin, Department of Respiratory Medicine, Affiliated Shanghai Sixth People's Hospital, Shanghai Jiao Tong University, 600 Yishan Road, Shanghai 200233, P.R. China E-mail: yinshaojun2010@163.com

\section{${ }^{*}$ Contributed equally}

Abbreviations: TF-3, theaflavin-3,3'-digallate; CSE, cigarette smoke extract; RIPK3, receptor-interacting serine/threonine-protein kinase three; MLKL, mixed lineage kinase domain-like; ROS, reactive oxygen species

Key words: TF-3, emphysema, CSE of phosphorylated (p)-RIPK3 and phosphorylated (p-)MLKL, which indicated that TF-3 suppressed necroptosis via the p38 MAPK/RIPK3/MLKL signaling pathways. In vivo, it was observed that TF-3 treatment significantly attenuated morphological lung injury in mice with CSE-induced emphysema. Moreover, TF-3 significantly reduced the levels of proinflammatory cytokines, TNF- $\alpha$ and IL- $1 \beta$ and significantly enhanced the antioxidant capacity of the lung tissues in mice with emphysema. TF-3 also significantly inhibited the levels of p-RIPK3 and p-MLKL in the lungs of mice with emphysema. Therefore, the present study indicated that TF-3 may attenuate CSE-induced emphysema in mice by inhibiting necroptosis.

\section{Introduction}

Chronic obstructive pulmonary disease (COPD) is a common, preventable and treatable disease characterized by persistent respiratory symptoms and progressive airflow obstruction documented on spirometry; it is associated with an abnormal inflammatory response of the lungs to noxious particles or gases (1). COPD affects $\sim 400$ million individuals and is already the third leading cause of death worldwide, which the World Health Organization predicted would not occur until 2030 (1). Pulmonary emphysema is one of the most important pathological manifestations of COPD, and the pathogenesis of pulmonary emphysema includes non-specific inflammation propagated by cigarette smoke injury, increased oxidative stress, imbalanced proteases/anti-proteases and subsequent alveolar cell apoptosis, which results in the histological hallmark of enlarged alveolar spaces (2).

Necroptosis is a mechanism of genetically programmed lytic cell death that is considered to serve a role in the destruction of pathogen-infected cells and/or dysfunctional cells during certain degenerative or inflammatory disorders. Necroptosis can be triggered by multiple innate immune signaling pathways that lead to the phosphorylation and activation of the necroptotic kinase, receptor-interacting serine/threonine-protein kinase (RIPK3). RIPK3 activates the mixed lineage kinase domain-like protein (MLKL) via phosphorylation, which causes substantial conformational changes that enables the trafficking of MLKL to the plasma membrane, 
where it induces membrane permeabilization (3). It has previously been reported that that necroptosis may contribute to the development of pulmonary emphysema (4). However, the exact molecular mechanism remains largely unknown. Therefore, studying the role of necroptosis in the pathogenesis of pulmonary emphysema is of vital importance.

Black tea, one of the most common types of tea, is made of fresh green tea leaves fermented and oxidized by polyphenol oxidase. This process converts a large proportion of catechins into theaflavins and thearubigins (5). The theaflavins of black tea include theaflavin (TF-1), theaflavin-3-gallate (TF-2a), theaflavin-3'-gallate (TF-2b) and theaflavin-3,3'-digallate (TF-3). A previous study has demonstrated that theaflavins, especially TF-3, attenuate oxidative stress and inflammation (6). TF-3 also has been indicated to decrease the incidence of coronary heart disease, exhibit an advantageous effect on the bone mineral density and the potential to prevent cancer (7-9). However, the effect of TF-3 in protecting against cigarette smoke extract (CSE)-induced pulmonary emphysema and the mechanism of action remains unclear. It can therefore be hypothesized that drinking black tea may prevent CSE-induced pulmonary emphysema. The aim of the present study was to investigate the protective effect of TF-3 on pulmonary emphysema in CSE-treated mice via the necroptotic signaling pathway.

\section{Materials and methods}

Ethics statement. All animal procedures and experiments were approved by the Institutional Animal Care and Use Committee of Shanghai Jiao Tong University (Shanghai, China). The experiments were performed following the Guide for the Care and Use of Laboratory Animals published by the National Institutes of Health, 8th edition (10).

Drugs and antibodies. TF-3, benzyloxycarbonyl-Val-AlaAsp-fluoromethylketone (z-VAD), necrostatin-1 and SB203580 were purchased from MedChemExpress. All antibodies were purchased from Cell Signaling Technology, Inc. The following primary antibodies were used: phosphorylated (p)-RIPK3 (1:1,000; cat. no. 93654), RIPK3 (1:1,000; cat. no. 13526), p-MLKL (1:1,000; cat. no. 91689), MLKL (1:1,000; cat. no. 14993), p-p38 MAPK (1:1,000; cat. no. 9211), p38 MAPK (1:1,000; cat. no. 9212), caspase-3 (1:1,000; cat. no. 9662) and GAPDH (1:1,000; cat. no. 2118). The secondary antibody used was HRP-conjugated goat anti-rabbit IgG (1:10,000; cat. no. 7074). Lipopolysaccharide (Escherichia coli 055:B5) was purchased from Sigma-Aldrich (Merck KGaA).

CSE preparation. The CSE was prepared using a previously described technique (11). Briefly, a cigarette (Marlboro; Philip Morris Products S.A.) was burned and the smoke was passed through a $0.22 \mu \mathrm{m}$ filter (MilliporeSigma) to remove particles and bacteria before being added to a vessel containing PBS ( $2 \mathrm{ml}$ per cigarette for mouse experiments) or DMEM (Gibco; Thermo Fisher Scientific, Inc.; $10 \mathrm{ml}$ per cigarette as $100 \%$ CSE used for cell experiments) using a vacuum pump. The reproducibility of the extract was assessed using an AB Sciex 3200 Qtrap mass spectrometer (SCIEX) connected to an
Agilent 1200 Series high-performance liquid chromatography (Agilent Technologies, Inc.). The following conditions were used: Chromatographic column, Diamonsil-C18 (length, $150 \mathrm{~mm}$; inner diameter, $4.6 \mathrm{~mm}$; particle size, $5 \mu \mathrm{m}$; Dikma Technologies, Inc.); flow rate, $0.6 \mathrm{ml} / \mathrm{min}$; mobile phase, phosphoric acid-citric acid buffer containing $30 \%(\mathrm{v} / \mathrm{v})$ acetonitrile [30 $\mathrm{mM} \mathrm{K}_{2} \mathrm{HPO}_{4}, 30 \mathrm{mM}$ citric acid, $0.5 \%$ (v/v) triethylamine, adjusted to $\mathrm{pH} 6.7$ with $10 \mathrm{M} \mathrm{NaOH}$ ]; column temperature, $25^{\circ} \mathrm{C}$; sample quantity, $20 \mu \mathrm{l}$; internal standard, the nicotine standard (cat. no. N0267; Sigma-Aldrich; Merck KGaA) was dissolved in methanol to prepare the experimental standard $(10 \mathrm{mg} / \mathrm{ml})$. The ionization mode was positive ion mode, the source temperature was $700^{\circ} \mathrm{C}$ and the nebulizer pressure was 50 psi. The $\mathrm{pH}$ of the CSE-PBS/DMEM solution was 7.2-7.4 and the solution was freshly prepared for each experiment.

Cell culture and treatment. The human normal lung epithelial BEAS-2B cell line was purchased from the American Type Culture Collection (cat. no. CRL-9609 ${ }^{\mathrm{TM}}$ ) and maintained in DMEM supplemented with 10\% FBS (cat. no. 10270-106; Gibco; Thermo Fisher Scientific, Inc.) and 1\% vol/vol streptomycin/penicillin (Gibco; Thermo Fisher Scientific, Inc.) in an incubator at $37^{\circ} \mathrm{C}$ with $5 \% \mathrm{CO}_{2}$. BEAS-2B cells were pretreated with TF-3 $(5-20 \mu \mathrm{M})$ for $24 \mathrm{~h}$ at $37^{\circ} \mathrm{C}$ before the CSE challenge. The pan-caspase inhibitor z-VAD $(20 \mu \mathrm{M})$, the necroptosis inhibitor necrostatin-1 $(20 \mu \mathrm{M})$ or the p38 MAPK inhibitor SB203580 $(0.5 \mu \mathrm{M})$ were preincubated with the cells for $1 \mathrm{~h}$ at $37^{\circ} \mathrm{C}$ before the CSE challenge to stimulate necroptosis, inhibit necroptosis or inhibit p38 MAPK, respectively. For all drug treatments, cells were maintained in DMEM supplemented with $0.2 \% \mathrm{FBS}$ at $37^{\circ} \mathrm{C}$. The dose and treatment duration of all drugs were selected according to previous studies (7,12-14).

Cell viability. Cell viability was determined using the Cell Counting Kit-8 (CCK-8; Dojindo Laboratories, Inc.) assay. In brief, BEAS-2B cells $\left(2.5 \times 10^{4}\right.$ cells $\left./ \mathrm{ml}\right)$ were seeded into 96-well plates and incubated overnight at $37^{\circ} \mathrm{C}$. The cells were treated with $\operatorname{CSE}(1,5,10,20$ or $50 \%)$ alone, TF-3 $(5,10$ or $20 \mu \mathrm{M})$ alone or CSE $(10 \%)$ in combination with TF-3 (5, 10 or $20 \mu \mathrm{M})$. Following stimulation, $10 \mu \mathrm{l}$ of CCK-8 reagent was added to each well and then incubated for $2 \mathrm{~h}$ at $37^{\circ} \mathrm{C}$. The absorbance of each well was measured at $450 \mathrm{~nm}$ using a microplate reader (Molecular Devices, LLC). The viability of the treated cells was calculated as follows: (absorbance $_{450 \mathrm{~nm}}$ of the therapeutic group/absorbance $450 \mathrm{~nm}$ of the control group) $\mathrm{x} 100 \%$.

LDH assay. Lactate dehydrogenase was determined using an LDH Assay Kit (Beyotime Institute of Biotechnology). In brief, BEAS-2B cells ( $\sim 80 \%$ cell confluence) treated with CSE (10\%) and/or TF-3 $(20 \mu \mathrm{M})$ were incubated with $10 \mu \mathrm{l} \mathrm{LDH}$ releasing reagent for $1 \mathrm{~h}$ at $37^{\circ} \mathrm{C}$. The samples were centrifuged $\left(400 \mathrm{x} \mathrm{g} ; 5 \mathrm{~min} ; 25^{\circ} \mathrm{C}\right)$ and the $\mathrm{LDH}$ production levels of the supernatant were detected according to the manufacturer's instructions.

ROS generation assay. ROS generation was determined using a ROS Assay Kit (Beyotime Institute of Biotechnology). In brief, BEAS-2B cells ( $\sim 80 \%$ cell confluence) treated with 
CSE (10\%) and/or TF-3 $(20 \mu \mathrm{M})$ were incubated with $10 \mu \mathrm{M}$ 2',7'-dichlorodihydrofluorescein diacetate for $30 \mathrm{~min}$ at $37^{\circ} \mathrm{C}$ in the dark. The green fluorescence intensity was determined using a fluorescence microscope (Leica Microsystems GmbH; magnification, x200) and analyzed using ImageJ v1.8.0 software (National Institutes of Health).

$R N A$ extraction and reverse transcription-quantitative $P C R$ $(R T-q P C R)$. The mRNA expression levels of proinflammatory cytokines were determined using RT-qPCR. In brief, BEAS-2B cells treated with CSE (10\%) and/or TF-3 (20 $\mu \mathrm{M})$ and were lysed with TRIzol ${ }^{\circledR}$ reagent (Thermo Fisher Scientific, Inc.). mRNA was quantified using an ultraviolet spectrophotometer and subsequently, $1 \mu \mathrm{g}$ of RNA was reverse transcribed into cDNA using a HiScript ${ }^{\circledR}$ II Q RT SuperMix kit (Vazyme Biotech Co., Ltd.) at $50^{\circ} \mathrm{C}$ for $15 \mathrm{~min}$ and then at $85^{\circ} \mathrm{C}$ for $5 \mathrm{sec}$. qPCR was performed using ChamQ Universal SYBR qPCR Master Mix (Vazyme Biotech Co., Ltd.) and a deep-well qPCR detection system (LightCycler 480; Roche Diagnostics) using the following thermocycling conditions: Initial denaturation at $95^{\circ} \mathrm{C}$ for $5 \mathrm{~min}$; followed by 35 cycles of denaturation at $95^{\circ} \mathrm{C}$ for $60 \mathrm{sec}$, annealing at $58^{\circ} \mathrm{C}$ for $60 \mathrm{sec}$ and extension at $72^{\circ} \mathrm{C}$ for $50 \mathrm{sec}$. The primers used for qPCR were as follows: IL- 6 forward (F), 5'-CACTGGTCTTTTGGAGTTTGAG-3' and reverse (R), 5'-GGACTTTTGTACTCATCTGCAC-3'; IL-1 $\beta$ F, 5'-AGCTACGAATCTCCGACCAC-3' and R, 5'CGTTAT CCCATGTGTCGAAGAA-3'; TNF- $\alpha$ F, 5'-GAGGCCAAG CCCTGGTATG-3' and R, 5'-CGGGCCGATTGATCTCAG C-3'; and GAPDH F, 5'-ACAACTTTGGTATCGTGGAAG G-3' and R, 5'-GCCATCACGCCACAGTTTC-3'. GAPDH was used as the internal reference gene. Quantification of mRNA expression levels was performed using the $2^{-\Delta \Delta \mathrm{Cq}}$ method (15). These data are presented as the relative mRNA expression level compared to that in control group, which was arbitrarily set to 1 .

Immunofluorescence microscopy for PI/DAPI staining. Necroptotic cells were identified using immunofluorescence. In brief, BEAS-2B cells were treated with CSE (10\%)/z-VAD $(20 \mu \mathrm{M})$ with either TF-3 $(20 \mu \mathrm{M})$ or necrostatin-1 $(20 \mu \mathrm{M})$, stained with PI for $30 \mathrm{~min}$ at $25^{\circ} \mathrm{C}$ in the dark $(5 \mu \mathrm{g} / \mathrm{ml}$; BD Biosciences), fixed in $3.7 \%$ paraformaldehyde for $10 \mathrm{~min}$ at $25^{\circ} \mathrm{C}$ and stained with DAPI (Sigma-Aldrich; Merck $\mathrm{KGaA}$ ) for $5 \mathrm{~min}$ at $25^{\circ} \mathrm{C}$ in the dark. Immunofluorescence images were captured using a fluorescence microscope (Leica Microsystems $\mathrm{GmbH}$ ). For each experiment, 100 cells were monitored with a fluorescence microscope and analyzed using ImageJ v1.8.0 software (National Institutes of Health).

Apoptosis assay. The necroptotic rate was determined using the Annexin V FITC Apoptosis Detection Kit I (BD Biosciences). In brief, BEAS-2B cells were treated with CSE (10\%)/z-VAD $(20 \mu \mathrm{M})$ with either TF-3 $(20 \mu \mathrm{M})$ or necrostatin-1 $(20 \mu \mathrm{M})$, washed twice with cold PBS and then resuspended in $1 \mathrm{X}$ binding buffer at a concentration of $1 \times 10^{6}$ cells $/ \mathrm{ml}$. The solution was subsequently transferred to a $5 \mathrm{ml}$ culture tube. A total of $5 \mu \mathrm{l}$ FITC Annexin V and $5 \mu \mathrm{l}$ PI were added, the cells were gently vortexed, and the samples were incubated for $15 \mathrm{~min}$ at room temperature $\left(25^{\circ} \mathrm{C}\right)$ in the dark. Cells were then diluted in $1 \mathrm{X}$ binding buffer $(400 \mathrm{ml})$ and analyzed using flow cytometry (BD FACSCelesta; BD Biosciences) and FlowJo software (v10.8.0; FlowJo LLC).

Western blotting. Protein expression levels of the relevant proteins were determined via western blotting. In brief, BEAS-2B cells treated with CSE $(10 \%) / \mathrm{z}-\mathrm{VAD}(20 \mu \mathrm{M})$ with either TF-3 (5-20 $\mu \mathrm{M})$ or SB203580 $(0.5 \mu \mathrm{M})$, were lysed in RIPA lysis buffer (Epizyme, Inc.) supplemented with a protease inhibitor and phosphatase inhibitor cocktail (Epizyme, Inc.). After the protein concentration was measured using a BCA protein assay kit (Epizyme, Inc.) and verified, the sample was mixed with $5 X$ SDS loading buffer. Total protein was separated using SDS-PAGE (20 $\mu \mathrm{g}$ protein/well) on an 8-12\% gel. Separated proteins were subsequently transferred to polyvinylidene difluoride membrane (MilliporeSigma), which were blocked with 5\% BSA (Sigma-Aldrich; Merck KGaA) in TBS with $0.1 \%$ Tween-20 (TBST) for $1 \mathrm{~h}$ at room temperature $\left(25^{\circ} \mathrm{C}\right)$. The membranes were immunoblotted with primary antibodies overnight at $4^{\circ} \mathrm{C}$. After washing with TBST three times, the membranes were incubated with secondary antibody for $1 \mathrm{~h}$ at room temperature $\left(25^{\circ} \mathrm{C}\right)$ and were then washed again with TBST three times. The membranes were scanned using Immobilon Western Chemiluminescent HRP Substrate (MilliporeSigma) and an imaging system (Amersham Imager 600; Cytiva). ImageJ v1.8.0 software (National Institutes of Health) was used for densitometry. GAPDH was used as the loading control. The data are presented as the relative protein expression level compared to the control group, which was arbitrarily set to 1 .

Experimental animal protocols. All studies involving animals were performed in compliance with the ARRIVE guidelines (16). All mice were housed at a constant room temperature of $22-23^{\circ} \mathrm{C}$ and humidity of $50-60 \%$ with an alternating $12 \mathrm{~h} \mathrm{light/dark} \mathrm{cycle} \mathrm{and} \mathrm{free} \mathrm{access} \mathrm{to} \mathrm{water} \mathrm{and}$ standard food. Twenty male C57BL/6J mice maintained in the animal facility (20-25 g body weight; specific pathogen free) of Shanghai Jiao Tong University were randomly selected and divided into the following four groups at 7 weeks of age: i) Control; ii) TF-3; iii) CSE; and iv) CSE + TF-3 ( $n=5$ each group). The total experimental period was four weeks. The control group was intraperitoneally (i.p.) injected with vehicle (normal saline), the TF-3 group was given TF-3 $(5 \mathrm{mg} / \mathrm{kg}$ body weight) by oral gavage daily for 28 days and the CSE group was injected with $0.3 \mathrm{ml}$ of CSE/PBS on day 0,11 and 22 . The $\mathrm{CSE}+\mathrm{TF}-3$ group was i.p. injected with $0.3 \mathrm{ml}$ of CSE on day 0,11 and 22 along with TF-3 (5 mg/kg body weight). The doses and administration routes of CSE and TF-3 were based on previous studies $(6,11,17)$.

ELISA assays. TNF- $\alpha$ (cat. no. DY410; Mouse TNF-alpha DuoSet ELISA) and IL-1 $\beta$ (cat. no. DY401; Mouse IL-1 beta/IL-1F2 DuoSet ELISA) levels in the lung tissue were measured with commercially available ELISA kits (Novus Biologicals, LLC) according to the manufacturer's instructions.

Malondialdehyde (MDA), superoxide dismutase (SOD) and glutathione (GSH) assays. The lung homogenate was dissolved in extraction buffer (cell lysis buffer; Beyotime Institute of Biotechnology) to detect MDA (cat. no. A003-1-2; 
A

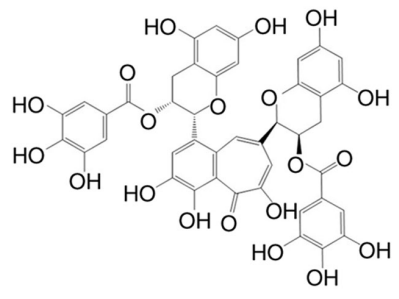

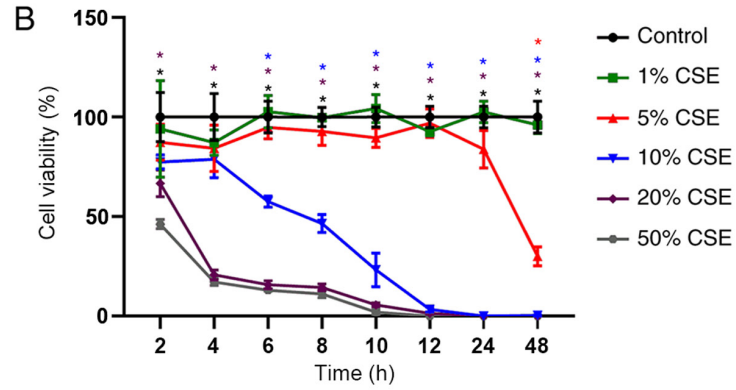
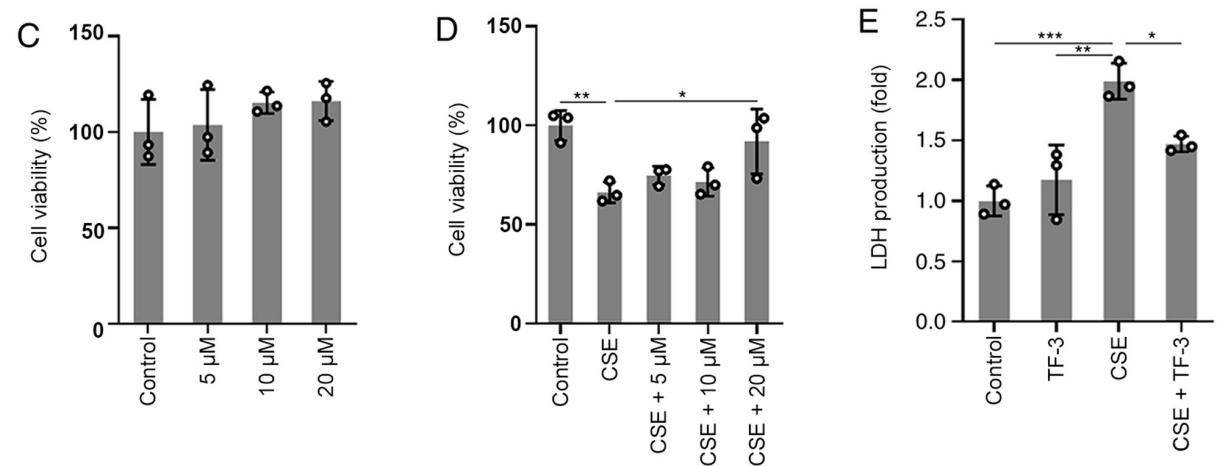

Figure 1. TF-3 attenuates the cytotoxicity of CSE on BEAS-2B cells. (A) Chemical structure of TF-3. (B) Changes in BEAS-2B cell viability induced by the various concentrations of CSE (1-50\%) at different time points (2-48 h). ${ }^{*} \mathrm{P}<0.05$ vs. control. (C) Effects of TF-3 on cell viability. Cells were treated with TF-3 $(5-20 \mu \mathrm{M})$. (D) Cells were treated with CSE (10\%) for $6 \mathrm{~h}$ alone or in combination with TF-3 (5-20 $\mu \mathrm{M})$ pretreatment. (E) Effects of TF-3 on LDH production. Cells were treated with CSE (10\%) for $6 \mathrm{~h}$ alone, TF-3 $(20 \mu \mathrm{M})$ for $24 \mathrm{~h}$ alone or CSE (10\%) for $6 \mathrm{~h}$ in combination with TF-3 (20 $\mu \mathrm{M})$ pretreatment. Data are presented as the mean \pm SEM of at least three independent experiments indicated by dots. One-way ANOVA was performed followed by Tukey's post hoc test for statistical comparisons between more than two groups. ${ }^{*} \mathrm{P}<0.05,{ }^{* *} \mathrm{P}<0.01$ and ${ }^{* * *} \mathrm{P}<0.001$. CSE, cigarette smoke extract; TF-3, theaflavin-3,3'-digallate.

MDA Assay Kit), SOD (cat. no. A001-3-2; SOD Assay Kit) and GSH (cat. no. A006-2-1; GSH Assay Kit) levels using commercially available assay kits (Nanjing Jiancheng Bioengineering Institute), according to the manufacturer's instructions.

$H \& E$ staining. Mice were anesthetized by inhalation of isoflurane $(4 \%)$ and euthanized by exsanguination via right ventricle aspiration, followed by cervical dislocation. The left lungs of the mice were harvested and fixed with $4 \%$ paraformaldehyde at $25^{\circ} \mathrm{C}$ for $24 \mathrm{~h}$. After fixation, lung tissue was embedded in paraffin and sectioned at 5- $\mu \mathrm{m}$ thickness. The sections were stained with hematoxylin at $25^{\circ} \mathrm{C}$ for $10 \mathrm{~min}$ and eosin at $25^{\circ} \mathrm{C}$ for $3 \mathrm{~min}$ and visualized under a light microscope (magnification, $\mathrm{x} 400$ ). Emphysema was quantified by measuring the mean linear intercept and determining the destructive index (11).

Statistical analysis. All the data were normally distributed and are presented as the mean \pm SEM of three independent cell experiments or five independent animal experiments. Statistical analyses were performed using GraphPad Prism v8 software (GraphPad Software, Inc.). One-way ANOVA was performed followed by Tukey's post hoc test for comparisons among multiple groups. $\mathrm{P}<0.05$ was considered to indicate a statistically significant difference.

\section{Results}

TF-3 prevents CSE-induced cytotoxicity in BEAS- $2 B$ cells. In order to explore the cytotoxic effect of CSE and the protective effect of TF-3 (Fig. 1A) on BEAS-2B cells, CCK-8 assay was used to detect cell viability. The results of the concentration/time-response study, in which BEAS-2B cells were subjected to different concentrations of CSE (1-50\%) for up to $48 \mathrm{~h}$, are displayed in (Fig. 1B). The viability of BEAS-2B cells treated with 1 or 5\% CSE was not significantly different compared with the control cells before $24 \mathrm{~h}$. However, significant decreases in cell viability were observed at 20 or $50 \%$ CSE exposure compared with the control. Gradual decreases in cell viability were observed after exposure to $10 \% \mathrm{CSE}$ and cell viability was close to $58 \pm 3 \%$ at $6 \mathrm{~h}$. Therefore, cells were treated with $10 \%$ CSE for $6 \mathrm{~h}$ or vehicle as a control. No significant cytotoxicity of TF-3 (5-20 $\mu \mathrm{M})$ was observed, as displayed in (Fig. 1C). The results in (Fig. 1D) demonstrated that TF-3 significantly reversed the decrease in viability of the CSE-induced BEAS-2B cells compared with the CSE group. Moreover, the membrane integrity of the cells was evaluated via the LDH assay. TF-3 significantly reduced the production of LDH released into the medium (Fig. 1E) in CSE-induced BEAS-2B cells compared with the CSE group.

TF-3 reduces ROS generation and $m R N A$ expression levels of TNF- $\alpha, I L-6$ and IL-1 $\beta$ in CSE-treated BEAS- $2 B$ cells. To further understand the role of TF-3 in ROS and inflammatory cytokine generation, BEAS-2B cells were pretreated with TF-3 $(20 \mu \mathrm{M})$ for $24 \mathrm{~h}$ prior to CSE challenge. The results displayed in (Fig. 2A and B) demonstrated that CSE-induced ROS generation was significantly suppressed by TF-3 compared with the CSE group, whereas TF-3 treatment on healthy cells did not affect ROS generation compared with the control. The mRNA expression levels of TNF- $\alpha$, IL- 6 and IL-1 $\beta$ were detected using RT-qPCR. As displayed in (Fig. 2C-E), the mRNA expression levels of TNF- $\alpha$, IL-1 $\beta$ 
A
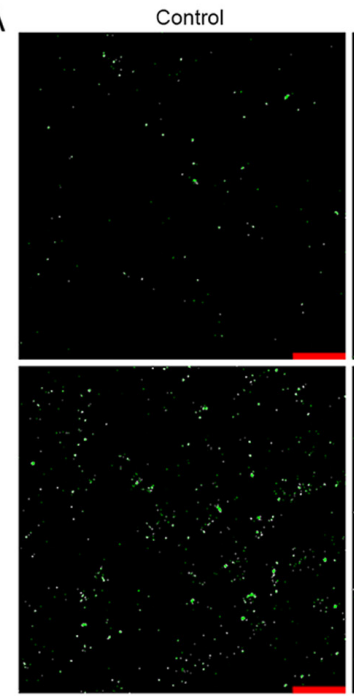

CSE

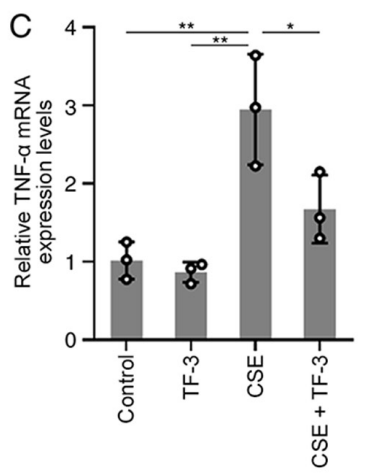

TF-3
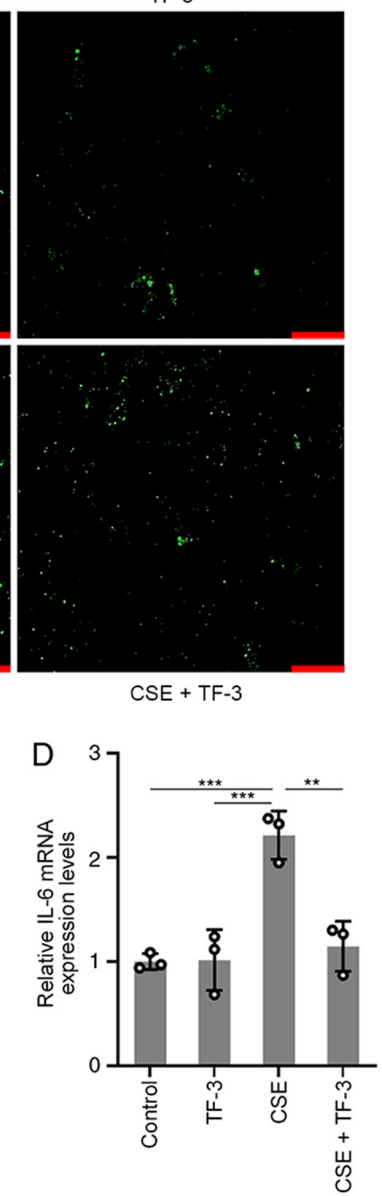
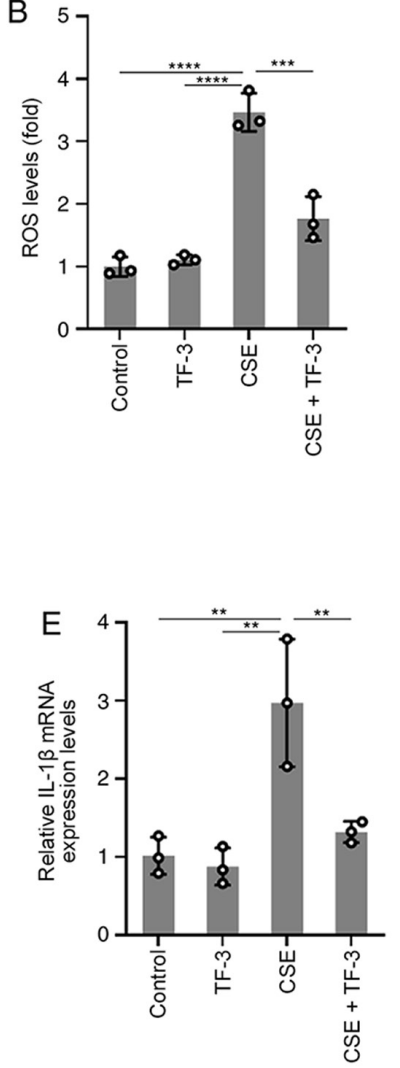

Figure 2. Effects of TF-3 on ROS generation and TNF- $\alpha$, IL-6 and IL-1 $\beta$ mRNA expression levels. Cells were treated with CSE (10\%) for 6 h with or without TF-3 $(20 \mu \mathrm{M})$ pretreatment. (A) ROS generation was determined using a fluorescence microscope. Scale bars, $50 \mu \mathrm{m}$. (B) Quantification of ROS generation. mRNA expression levels were measured using reverse transcription-quantitative PCR using GAPDH as a reference gene. Quantification of the relative mRNA expression levels of (C) TNF- $\alpha$, (D) IL-6 and (E) IL-1 $\beta$. Data are presented as the mean \pm SEM of three independent experiments indicated by dots. One-way ANOVA was performed followed by Tukey's post hoc test for statistical comparisons among more than two groups. ${ }^{*} \mathrm{P}<0.05,{ }^{* *} \mathrm{P}<0.01,{ }^{* * *} \mathrm{P}<0.001$ and ${ }^{* * * * *} \mathrm{P}<0.0001$. CSE, cigarette smoke extract; TF-3, theaflavin-3,3'-digallate; ROS, reactive oxygen species.

and IL-6 in CSE-induced BEAS-2B cells were significantly diminished by TF-3 treatment compared with the CSE only group. These results indicated that TF-3 may serve a crucial role in antioxidation and anti-inflammation.

TF-3 inhibits nonapoptotic cell death in CSE $/ z$-VAD-treated $B E A S-2 B$ cells. The effect of TF-3 on necroptosis was examined. The necroptotic rate was determined using immunofluorescence and flow cytometry. Necrostatin-1, an inhibitor of necroptosis, was used to examine whether CSE/z-VAD induced apoptosis. The positive rate of PI was significantly increased following necroptotic stimulation in the immunofluorescence assay compared with the control group, whereas PI-positive cells were significantly decreased by Necrostatin-1 compared with the CSE/z-VAD only group, which indicated that CSE/z-VAD induced necroptosis in BEAS-2B cells. Furthermore, TF-3 decreased the number of PI-positive cells in CSE/z-VAD-treated cells compared with the CSE/z-VAD only group (Fig. 3A and B). Following necroptotic stimulation, the necrotic or late apoptotic (Annexin $\mathrm{V}+/ \mathrm{PI}+$ in $\mathrm{Q} 2$ ) rates were increased. By contrast, no obvious elevation of early apoptotic (Annexin V+/PI- in Q4) rates was observed. Similar effects of necrostatin-1 and TF-3 were observed in the flow cytometry assay, and they both reduced the rates of cells in the area Q2 compared with the CSE/z-VAD only group, which indicated that TF-3 decreased the necroptotic rates of CSE/z-VAD-treated cells (Fig. 3C and D). To further exclude the role of TF-3 in necroptosis induced by CSE/z-VAD, caspase-3 protein expression levels were examined. CSE/z-VAD treatment did not significantly affect caspase-3 activities in BEAS-2B cells (Fig. 4A and E). Furthermore, no cleavage of caspase-3 was detected via western blotting analysis (Fig. 4A) indicating that apoptosis was not responsible for $\mathrm{CSE} / \mathrm{z}-\mathrm{VAD}$-induced cell death in BEAS-2B cells. These results suggested that TF-3 may inhibit CSE/z-VAD-induced nonapoptotic cell death in BEAS-2B cells.

TF-3 suppresses necroptosis via the p38 MAPK/RIPK3/MLKL signaling pathway in CSE $/ z-V A D$-induced BEAS-2B cells. To investigate the effect of TF-3 on necroptosis at the protein expression level of RIPK3 and MLKL, these key mediators of the necroptosis pathway were investigated via western blotting. As demonstrated in (Fig. 4A, C and D), western blotting determined that the protein expression levels of p-RIPK3 and p-MLKL were significantly increased following CSE/z-VAD treatment compared with the control. However, TF-3 treatment significantly decreased the protein expression levels 
A
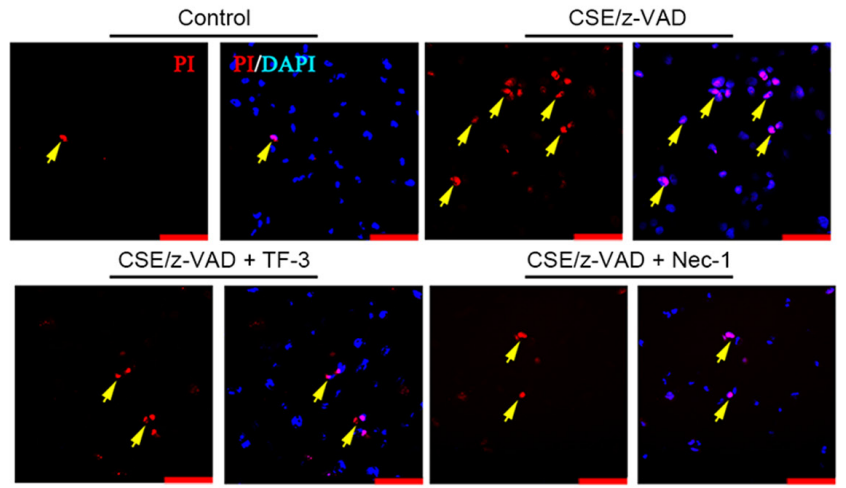

C
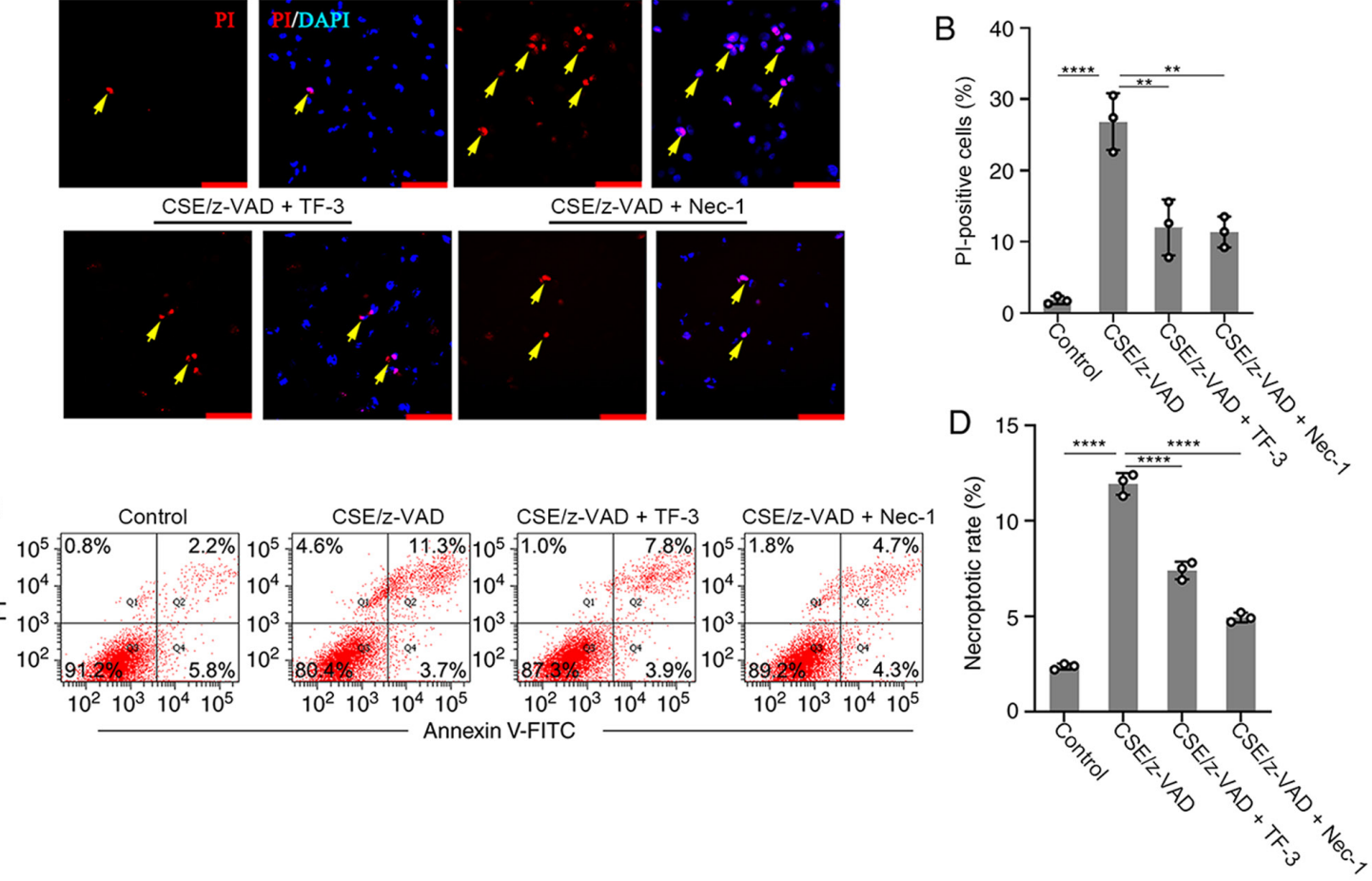

Figure 3. Effects of TF-3 on necroptosis. BEAS-2B cells were treated with CSE $(10 \%) / \mathrm{z}-\mathrm{VAD}(20 \mu \mathrm{M})$ alone or in combination with either TF-3 (20 $\mu \mathrm{M})$ or necrostatin-1 $(20 \mu \mathrm{M})$ and were stained with PI and DAPI. (A) For each experiment 100 cells were monitored with a fluorescence microscope. Yellow arrows indicate necroptosis. Scale bars, $20 \mu \mathrm{m}$. (B) Quantification of PI positive cells. (C) Cells were stained with PI and Annexin V-FITC and analyzed via flow cytometry. (D) Necroptotic rate of cells. Data are presented as the mean \pm SEM of three independent experiments indicated by dots. One-way ANOVA was performed followed by Tukey's post hoc test for statistical comparisons among more than two groups. ${ }^{* *} \mathrm{P}<0.01,{ }^{* * * * *} \mathrm{P}<0.0001$. CSE, cigarette smoke extract; TF-3, theaflavin-3,3'-digallate; Nec-1, necrostatin-1; PI, propidium iodide.
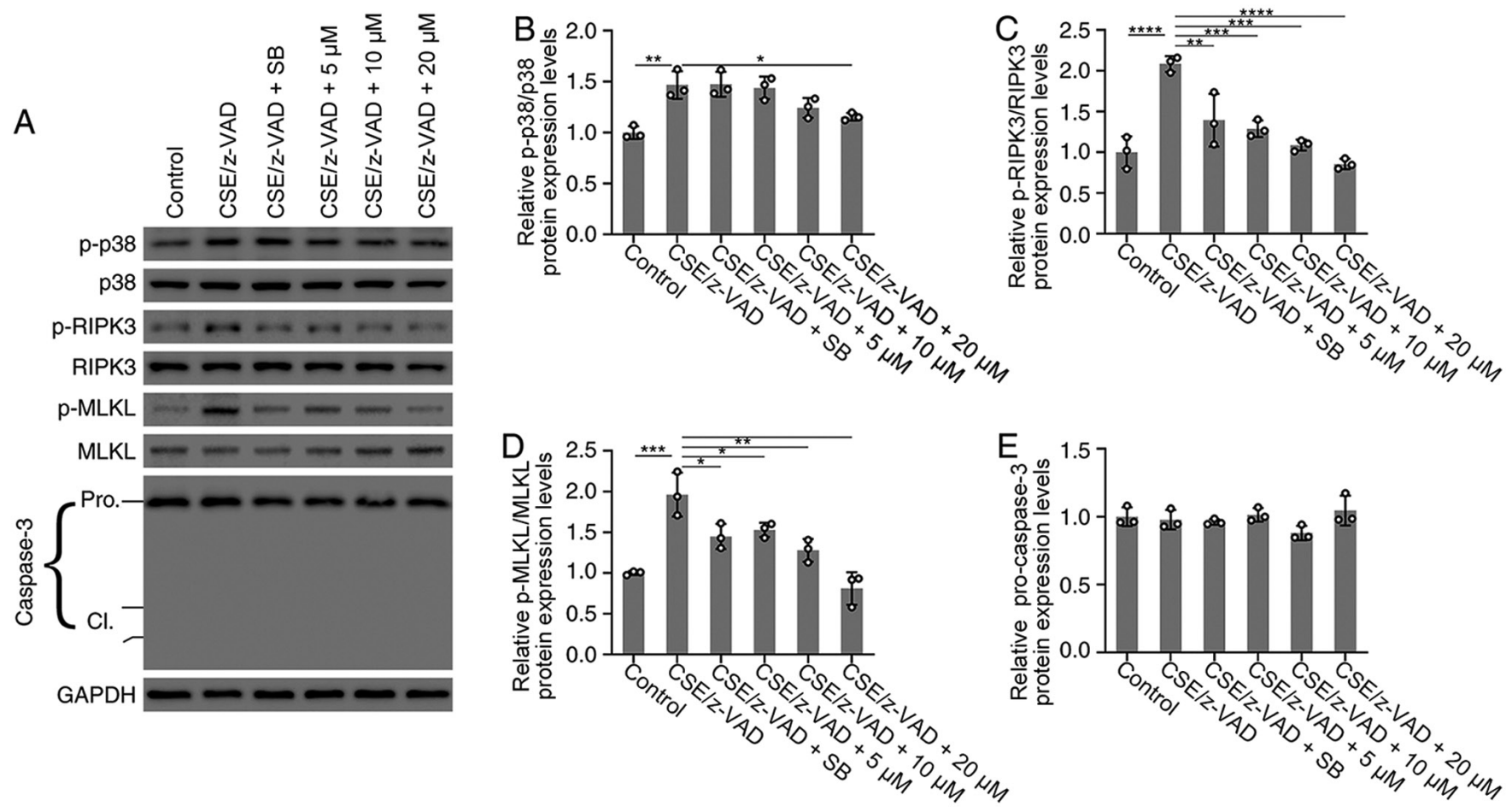

Figure 4. Effects of TF-3 on the protein expression levels of p38 MAPK, RIPK3, MLKL and caspase-3. BEAS-2B cells were treated with CSE (10\%)/z-VAD $(20 \mu \mathrm{M})$ alone or in combination with either TF-3 $(5-20 \mu \mathrm{M})$ or SB203580 $(0.5 \mu \mathrm{M})$. (A) Protein expression levels of p-p38 MAPK/p38 MAPK, p-RIPK3/RIPK3, p-MLKL/MLKL and caspase-3 were determined via western blotting with GAPDH as an internal control. Semi-quantification of the protein expression levels of (B) p-p38 MAPK, (C) p-RIPK3, (D) p-MLKL and (E) caspase-3. Data are presented as the mean \pm SEM of three independent experiments indicated by dots. One-way ANOVA was performed followed by Tukey's post hoc test for statistical comparisons among more than two groups. ${ }^{*} \mathrm{P}<0.05,{ }^{* * *} \mathrm{P}<0.01,{ }^{* * * *} \mathrm{P}<0.001$ and ${ }^{* * * * *} \mathrm{P}<0.0001$. CSE, cigarette smoke extract; TF-3, theaflavin-3,3'-digallate; RIPK3, receptor-interacting serine/threonine-protein kinase 3; MLKL, mixed lineage kinase domain-like; p, phosphorylated; $\mathrm{cl}$, cleaved. 

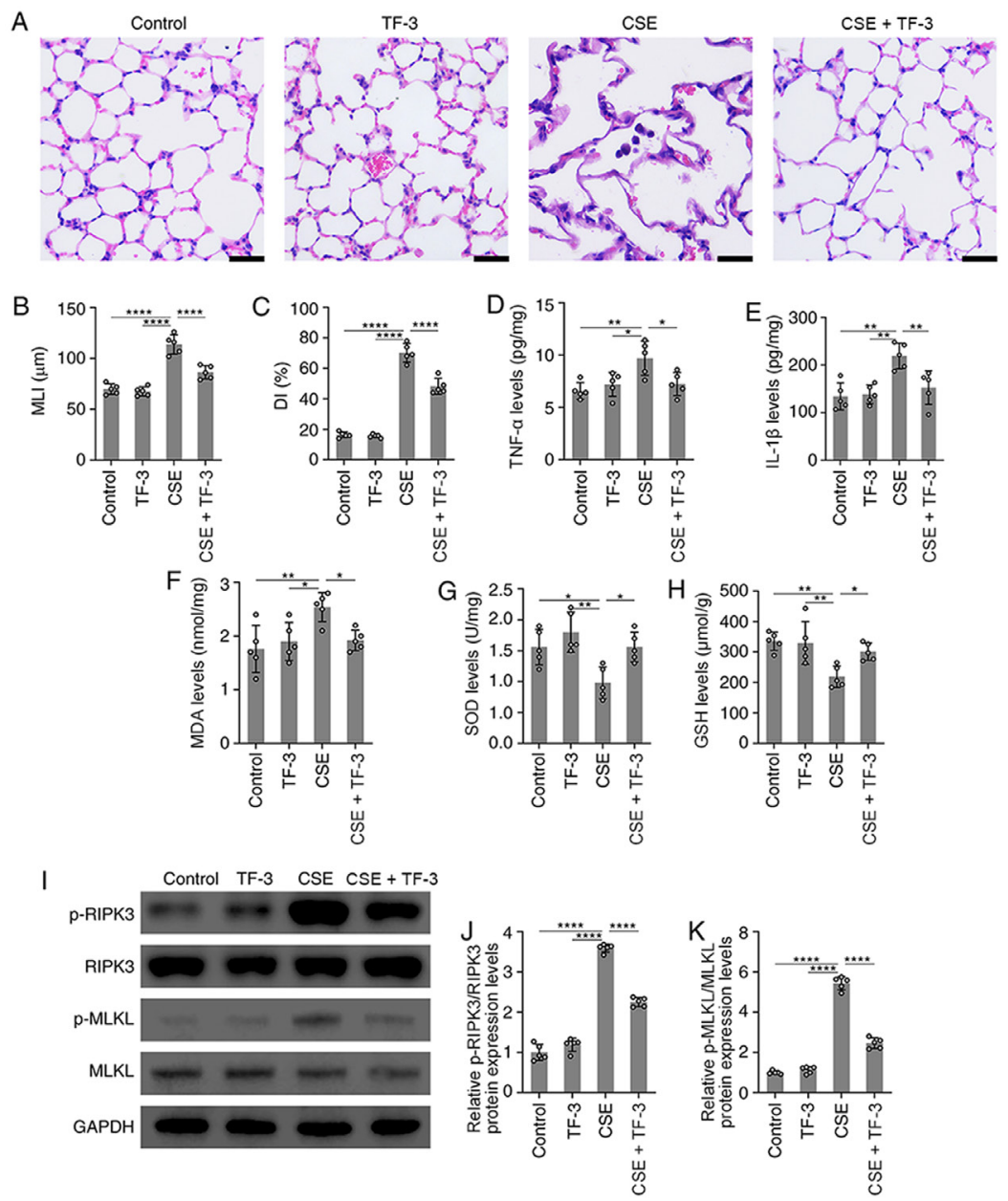

Figure 5. TF-3 attenuates emphysema in CSE-treated mice. (A) Images of lung tissue stained with H\&E. Scale bars, $20 \mu$ m. (B and C) Morphometric measurements of the MLI and DI in the four groups were quantified. (D and E) TNF- $\alpha$ and IL-1 $\beta$ levels in lung tissue were detected using ELISA and quantified. (F-H) MDA, SOD and GSH levels in lung tissue were detected with assay kits and quantified. (I) Protein expression levels of p-RIPK3/RIPK3 and p-MLKL/MLKL were determined via western blotting with GAPDH as an internal control. Semi-quantification of protein expression levels of (J) p-RIPK3 and $(\mathrm{K})$ p-MLKL were determined. Data are presented as the mean \pm SEM of five independent experiments indicated by dots. One-way ANOVA was performed followed by Tukey's post hoc test for statistical comparisons among more than two groups. ${ }^{*} \mathrm{P}<0.05$, ${ }^{* *} \mathrm{P}<0.01$ and ${ }^{* * * *} \mathrm{P}<0.0001$. MLI, mean linear intercept; DI, destructive index; MDA, malondialdehyde; SOD, superoxide dismutase; GSH, glutathione; RIPK3, receptor-interacting serine/threonine-protein kinase 3; MLKL, mixed lineage kinase domain-like; p, phosphorylated; CSE, cigarette smoke extract; TF-3, theaflavin-3,3'-digallate.

of p-RIPK3 and p-MLKL compared with the CSE/z-VAD group. Furthermore, the protein expression levels of $\mathrm{p} 38$ MAPK were examined. Western blotting demonstrated that TF-3 $(20 \mu \mathrm{M})$ significantly reduced the protein expression levels of p-p38 MAPK compared with the CSE/z-VAD group (Fig. 4A and B). Furthermore, the results demonstrated that SB203580, a specific inhibitor of the p38 MAPK signaling pathway, significantly reduced the level of p-RIPK3/p-MLKL in CSE/z-VAD-treated BEAS-2B cells, compared with the CSE/z-VAD only group. These results indicated that TF-3 may inhibit necroptosis via the p38 MAPK/RIPK3/MLKL signaling pathway in CSE/z-VAD-induced BEAS-2B cells.

TF-3 attenuates morphological lung injury in CSE-induced emphysema mice. In vivo, a mouse model of emphysema by i.p. injection of CSE within 4 weeks was established (17). Compared with the normal alveolar architecture of the control group, structural damage in the CSE group was observed, with an increase in alveolar size, broken alveolar wall and pulmonary emphysema. This indicated that the i.p. injection of CSE induced emphysema within 4 weeks. However, TF-3 markedly suppressed these emphysematous changes (Fig. 5A-C).

TF-3 suppresses oxidative stress and inflammatory responses in CSE-induced emphysema mice. Subsequently, the antioxidative and anti-inflammatory capacity of TF-3 in CSE-treated emphysema mice was explored. Compared with CSE only group, the results demonstrated that TF-3 significantly decreased TNF- $\alpha$, IL-1 $\beta$ and MDA levels but significantly increased the levels of SOD and GSH in the lungs (Fig. 5D-H). These results indicated that TF-3 may prevent CSE-induced emphysema by inhibiting oxidative stress and inflammatory responses.

TF-3 suppresses necroptosis in CSE-induced emphysema mice. The effect of TF-3 on necroptosis in CSE-induced emphysema mice was also detected. The western blotting results demonstrated that the protein expression levels of p-RIPK3 and p-MLKL were significantly increased in the lung tissue of CSE-treated mice compared with the control. 
However, TF-3 significantly decreased these protein expression levels compared with the CSE group (Fig. 5I-K).

\section{Discussion}

In the present study, the results demonstrated that TF-3 may exhibit a protective effect against emphysema in CSE-induced mice via the inhibition of necroptosis.

Black tea is a well-known beverage that is commonly consumed and contains theaflavins, which are considered to have anti-inflammatory and antioxidant effects (18). Among theaflavins, TF-3, which has two gallic acid moieties, exhibits the strongest anti-inflammatory and antioxidant activity (6).

Currently, all available evidence firmly suggests that oxidative stress modulated by ROS serves a significant role in the pathogenesis of pulmonary emphysema $(19,20)$. Under physiological conditions, ROS can help prevent pathological injury or noxious stimulation. However, excessive generation of ROS is thought to lead to cellular injury and oxidative stress (21). In the present study, the results demonstrated that antioxidant activity was significantly decreased in CSE-challenged BEAS-2B cells. However, TF-3 was capable of significantly blocking this decrease, which suggested that TF-3 may be involved in the pathophysiological pathway of oxidative stress in the development of emphysema.

Inflammation is a type of stress protection against tissue or cell damage induced by adverse external stimulation. A large number of proinflammatory cytokines are produced in the process of inflammation, including TNF- $\alpha$, IL-1 $\beta$ and IL-6, which are generally considered to be the most relevant proinflammatory cytokines in the inflammatory response (22). In the present study, TF-3 significantly reduced the mRNA expression levels of TNF- $\alpha$, IL- $1 \beta$ and IL-6 in CSE-induced BEAS-2B cells.

Increasing numbers of reports have shown that necroptosis, a novel nonapoptotic cell death mechanism, may contribute to the pathogenesis of pulmonary emphysema $(4,23-26)$. The difference between necrosis and necroptosis is that necrosis is a non-programmed and passive method of cell death, whereas necroptosis can be regulated (27). A previous study has also indicated that inhibition of the RIPK3/MLKL signaling pathway attenuates necroptosis (28). The results of the present study demonstrated that TF-3 significantly decreased the protein expression levels of p-RIPK3 and p-MLKL in CSE/z-VAD-treated BEAS-2B cells. Moreover, the flow cytometry and immunofluorescence assay results determined that TF-3 significantly suppressed the necroptosis stimulated by CSE/z-VAD. Collectively this evidence demonstrated that TF-3 may ameliorate CSE/z-VAD-induced inflammation and oxidative stress by inhibiting the RIPK3/MLKL-mediated necroptosis signaling pathway. Furthermore, it was confirmed that the p38 MAPK signaling pathway was strongly associated with necroptosis. SB203580, a specific inhibitor of p38 MAPK, was used to suppress the autophosphorylation and substrate phosphorylation of p38 MAPK (14). However, SB203580 does not inhibit the phosphorylation of p38 MAPK by upstream kinases. In CSE/z-VAD-treated BEAS-2B cells, the phosphorylation of RIPK3 and MLKL was significantly increased, but this was significantly ameliorated by SB203580, which indicated that p38 MAPK may promote RIPK3/MLKL-mediated necroptosis (Fig. 4A-D).
In vivo, it was observed that TF-3 significantly alleviated the emphysematous changes induced by CSE treatment in the lung tissue of mice, which indicated that TF-3 can inhibit emphysema morphologically. Furthermore, TF-3 may suppress inflammatory responses in the pathogenesis of emphysema and inflammatory cytokines (TNF- $\alpha$ and IL-1 $\beta$ ) were significantly reduced following TF-3 therapy. Moreover, the detection results of oxidative stress-related indicators (MDA, SOD and GSH) indicated that the antioxidant capacity of TF-3 may serve an important role in the inhibition of emphysema. Furthermore, the results of the present study demonstrated that necroptosis may be involved in the pathogenesis of emphysema by detecting the protein expression levels of p-RIPK3 and $\mathrm{p}$-MLKL in the lung tissue of CSE-induced mice. The results demonstrated that necroptosis was significantly inhibited by TF-3 in the lung tissue of CSE-induced emphysema mice. All this aforementioned evidence indicated that TF-3 may attenuate emphysema by suppressing necroptosis in CSE-induced mice.

The emphysema mouse model used in the present study has been previously described (17). The mechanism of this new method for establishing an animal model of emphysema is not well understood, but it has been reported to be related to the autoimmune mechanism of alveolar septal cell destruction (29). It is unclear whether the pathobiological mechanisms underlying modeling by CSE injection are the same as those of conventional inhalation of cigarette smoke for several months. However, following i.p. injection of CSE for four weeks, mice developed emphysema, which was accompanied by alveolar dilatation and destruction, and confirmed the efficiency of CSE injection in the establishment of a tobacco-related model of emphysema in mice. Furthermore, TF-3 suppressed these emphysematous changes. Therefore, these results suggested that TF-3 may inhibit the progression of pulmonary emphysema in CSE-induced mice.

In conclusion, the present study demonstrated that TF-3 attenuates lung tissue architecture damage, oxidative stress, inflammatory responses and necroptosis in the lung tissue of mice with CSE-induced pulmonary emphysema. Furthermore, it was indicated that TF-3 reduced ROS and proinflammatory cytokine generation and inhibited necroptosis via the p38 MAPK/RIPK3/MLKL signaling pathways in CSE-treated BEAS-2B cells. Thus, there may be signaling pathways that link oxidative stress, inflammation and necroptosis in the pathogenesis of emphysema. The evidence demonstrated that TF-3 serves an important role in protecting against pulmonary emphysema by alleviating oxidative stress, inflammation and necroptosis. However, the protective effect of TF-3 against pulmonary emphysema needs to be further explored. The current study still presents certain limitations, such as the lack of positive controls and the failure to explore the optimal concentration of TF-3. More experiments should be carried out to reveal the underlying mechanisms in vitro. Furthermore, the biological activity of TF-3 in other animal models, different treatment cycles and differences in its effect in humans and animals, need to be investigated.

\section{Acknowledgements}

Not applicable. 


\section{Funding}

This work was supported by a grant from the National Natural Science Foundation of China (grant no. 82004432). The funders had no role in the study design, in the collection, analysis and interpretation of data, in the writing of the report, or in the decision to submit the article for publication.

\section{Availability of data and materials}

The datasets used and/or analyzed during the current study are available from the corresponding author on reasonable request.

\section{Authors' contributions}

GL, ZZ and KW performed the experiments and analyzed the data. GL participated in the experimental design. GL and SY designed the study, analyzed the data and wrote the manuscript. GL and SY confirm the authenticity of all the raw data. All authors read and approved the final manuscript.

\section{Ethics approval and consent to participate}

The present study was conducted according to the revised Declaration of Helsinki and was approved by the Institutional Review Board and Animal Ethics Committee of Shanghai University of Medicine and Health Science (Shanghai, China). All the animal experiments were carried out following the National Institutes of Health Guide for the Care and Use of Laboratory Animals.

\section{Patient consent for publication}

Not applicable.

\section{Competing interests}

The authors declare that they have no competing interests.

\section{References}

1. Labaki WW and Rosenberg SR: Chronic obstructive pulmonary disease. Ann Intern Med 173: ITC17-ITC32, 2020.

2. Tuder RM, Yoshida T, Arap W, Pasqualini R and Petrache I: State of the art. Cellular and molecular mechanisms of alveolar destruction in emphysema: An evolutionary perspective. Proc Am Thorac Soc 3: 503-510, 2006.

3. Bedoui S, Herold MJ and Strasser A: Emerging connectivity of programmed cell death pathways and its physiological implications. Nat Rev Mol Cell Biol 21: 678-695, 2020.

4. Mizumura K, Cloonan SM, Nakahira K, Bhashyam AR, Cervo M, Kitada T, Glass K, Owen CA, Mahmood A, Washko GR, et al: Mitophagy-dependent necroptosis contributes to the pathogenesis of COPD. J Clin Invest 124: 3987-4003, 2014.

5. Balentine DA, Wiseman SA and Bouwens LC: The chemistry of tea flavonoids. Crit Rev Food Sci Nutr 37: 693-704, 1997.

6. Ukil A, Maity S and Das PK: Protection from experimental colitis by theaflavin-3,3'-digallate correlates with inhibition of IKK and NF-kappaB activation. Br J Pharmacol 149: 121-131, 2006.

7. Wu Y, Jin F, Wang Y, Li F, Wang L, Wang Q, Ren Z and Wang Y: In vitro and in vivo anti-inflammatory effects of theaflavin-3,3'-digallate on lipopolysaccharide-induced inflammation. Eur J Pharmacol 794: 52-60, 2017.

8. Ai Z, Wu Y, Yu M, Li J and Li S: Theaflavin-3, 3'-digallate suppresses RANKL-induced osteoclastogenesis and attenuates ovariectomy-induced bone loss in mice. Front Pharmacol 11: 803,2020
9. Pan H, Kim E, Rankin GO, Rojanasakul Y, Tu Y and Chen YC: Theaflavin-3, 3'-digallate inhibits ovarian cancer stem cells via suppressing Wnt/ $\beta$-Catenin signaling pathway. J Funct Foods 50: $1-7,2018$

10. Council NR: Guide for the Care and Use of Laboratory Animals: Eighth Edition. Washington, DC, The National Academies Press, 246, 2011.

11. Chen Y, Hanaoka M, Chen P, Droma Y, Voelkel NF and Kubo K: Protective effect of beraprost sodium, a stable prostacyclin analog, in the development of cigarette smoke extract-induced emphysema. Am J Physiol Lung Cell Mol Physiol 296: L648-L56, 2009.

12. Sun L, Wang H, Wang Z, He S, Chen S, Liao D, Wang L, Yan J, Liu W, Lei X and Wang X: Mixed lineage kinase domain-like protein mediates necrosis signaling downstream of RIP3 kinase. Cell 148: 213-227, 2012.

13. Sun W, Wu X, Gao H, Yu J, Zhao W, Lu JJ, Wang J, Du G and Chen X: Cytosolic calcium mediates RIP1/RIP3 complex-dependent necroptosis through JNK activation and mitochondrial ROS production in human colon cancer cells. Free Radic Biol Med 108: 433-444, 2017.

14. Cabrera J, Negrín G, Estévez F, Loro J, Reiter RJ and Quintana J: Melatonin decreases cell proliferation and induces melanogenesis in human melanoma SK-MEL-1 cells. J Pineal Res 49: 45-54, 2010.

15. Livak KJ and Schmittgen TD: Analysis of relative gene expression data using real-time quantitative PCR and the 2(-Delta Delta C(T)) method. Methods 25: 402-408, 2001.

16. Kilkenny C, Browne WJ, Cuthi I, Emerson M and Altman DG: Improving bioscience research reporting: The ARRIVE guidelines for reporting animal research. PLoS Biol 8: e1000412, 2010.

17. Zhang Y, Cao J, Chen Y, Chen P, Peng H, Cai S, Luo H and Wu SJ: Intraperitoneal injection of cigarette smoke extract induced emphysema, and injury of cardiac and skeletal muscles in BALB/C mice. Exp Lung Res 39: 18-31, 2013.

18. Bahramsoltani R, Ebrahimi F, Farzaei MH Baratpourmoghaddam A, Ahmadi P, Rostamiasrabadi P, Rasouli Amirabadi AH and Rahimi R: Dietary polyphenols for atherosclerosis: A comprehensive review and future perspectives. Crit Rev Food Sci Nutr 59: 114-132, 2019.

19. Tuder RM and Petrache I: Pathogenesis of chronic obstructive pulmonary disease. J Clin Invest 122: 2749-2755, 2012.

20. Polverino F, Rojas-Quintero J, Wang X, Petersen H, Zhang L, Gai X, Higham A, Zhang D, Gupta K, Rout A, et al: A disintegrin and metalloproteinase Domain-8: A novel protective proteinase in chronic obstructive pulmonary disease. Am J Respir Crit Care Med 198: 1254-1267, 2018.

21. Yang CS, Kim JJ, Lee SJ, Hwang JH, Lee CH, Lee MS and Jo EK: TLR3-triggered reactive oxygen species contribute to inflammatory responses by activating signal transducer and activator of transcription-1. J Immunol 190: 6368-6377, 2013.

22. Martinon F: Signaling by ROS drives inflammasome activation. Eur J Immunol 40: 616-619, 2010.

23. Galluzzi L, Kepp O, Chan FK and Kroemer G: Necroptosis: Mechanisms and relevance to disease. Annu Rev Pathol 12: 103-130, 2017.

24. Pearson JS, Giogha C, Mühlen S, Nachbur U, Pham CL, Zhang Y, Hildebrand JM, Oates CV, Lung TW, Ingle D, et al: EspL is a bacterial cysteine protease effector that cleaves RHIM proteins to block necroptosis and inflammation. Nat Microbiol 2: 16258, 2017.

25. Wang Y, Liu J, Zhou JS, Huang HQ, Li ZY, Xu XC, Lai TW, Hu Y, Zhou HB, Chen HP, et al: MTOR suppresses cigarette smoke-induced epithelial cell death and airway inflammation in chronic obstructive pulmonary disease. J Immunol 200: 2571-2580, 2018.

26. Ryter SW, Rosas IO, Owen CA, Martinez FJ, Choi ME, Lee CG, Elias JA and Choi AMK: Mitochondrial Dysfunction as a pathogenic mediator of chronic obstructive pulmonary disease and idiopathic pulmonary fibrosis. Ann Am Thorac Soc 15 (Suppl 4): S266-S272, 2018.

27. Newton K and Manning G: Necroptosis and Inflammation. Annu Rev Biochem 85: 743-763, 2016.

28. Weinlich R, Oberst A, Beere HM and Green DR: Necroptosis in development, inflammation and disease. Nat Rev Mol Cell Biol 18: 127-136, 2017.

29. Sullivan AK, Simonian PL, Falta MT, Cosgrove GP, Brown KK, Kotzin BL, Voelkel NF and Fontenot AP: Activated oligoclonal CD4+ T cells in the lungs of patients with severe emphysema. Proc Am Thorac Soc 3: 486, 2006. 